\title{
Intelligent backpack weight assessment system
}

\author{
Diana Marie M. Bulatao, Precious Mae V. Carolino, Aizel Cate S. Equipaje, \\ Cristine Jin DS. Estrada, Paolo Adrian D. Papa, E Edison A. Roxas* \\ Department of Electronics Engineering, Faculty of Engineering \\ University of Santo Tomas, 1015 Manila, Philippines
}

\begin{abstract}
Overloaded backpacks have been shown to be associated with the tendency of having back, neck, shoulder pains, posture problems and even musculoskeletal disorders. A design for a backpack system is presented that will alleviate these negative effects. The backpack system is equipped with sensors and microprocessors that will alert the user when the load exceeds his maximum weight bearing capacity. The system will automatically obtain the anthropometric measurements of the user and determine from these data the acceptable load, which has been suggested to be 10 to $15 \%$ of the user's body weight.
\end{abstract}

Keywords: backpack load, intelligent system, maximum weight bearing capacity

\section{INTRODUCTION}

From a canvas bag in the 1920's to a limitededition backpack featuring solar panels, it is evident how backpacks have technologically advanced to compensate the additional demands of consumers [1]. Granted that there are cell phones and iPod compartments, and padded sleeves for laptops, as well as air cushioning in the straps, there are other issues which concerns overloading that although recognized and addressed, a serious monitoring and control should be implemented for the prevention of long term health problems.

Several studies have shown a considerable relationship between backpack weight and the occurrence of health-related effects especially to the students since their bodies are still being

*To whom correspondence should be addressed earoxas@ust.edu.ph emergent and developing; therefore, they are more vulnerable to injury. With the strongest muscles in the back and in the abdomen, it is possible to support backpacks with excessive weights. However, it is alarming that year after year, U.S. Consumer Product Safety Commission reports 5,500 Americans suffering from backpack-related injuries and that 64 percent of those diagnosed are children [2].

A study led by Manila Doctor's Hospital have provided statistics indicating that $54 \%$ of the grade 5-6 elementary students from selected schools in Metro Manila have been found out to be carrying backpacks of weight more than $10 \%$ of their body mass. From these group, 23\% of which are identified as having pain in the back [3]. A noticeable effect of excessive loading of backpack is when there is a need for a child to bend forward [4]. Low back pains or degenerative disorders due to carrying loads with bent back 
are brought about by bearing loadings over extended time [5].

As a result, experts suggest that the suitable weight of backpacks should be, at maximum, 10 $15 \%$ of a person's body weight. It is scientifically proven that going over this value will induce the occurrence of neck and back pain. Cases of such discomforts have been the complaint of $10-30 \%$ of healthy children who have experienced these in their teenage years [6].

Efforts to prevent backpack injuries emerge to reduce the number of affected individuals. First, the solution is basically about eliminating the need to carry the backpack such as by adding rollers or wheels so that it can be pulled rather than to be carried. It is certainly helpful but in real practice, this method has been less than effective because of reluctance of many, particularly the adolescents, to use this wheeled-feature backpack. Furthermore, the backpack usually has to be lifted at some point such as when the student will ride to a vehicle or when the pathway of the student is not appropriate for the wheels to be used. Then, there comes the additional structures of the backpack such as wide, padded shoulder straps, and belt to be fastened at the abdomen and/or hip.

As these attempts are unsuccessful, researchers continue to conduct study with regard to the prevention of backpack injuries until the use of technology arise. The present innovation includes studies about equipping the backpack with a weight assessment system using mechanical or electrical devices [7].

This paper describes a design for a backpack system equipped with sensors and microprocessors that will alert the user when the load exceeds his maximum weight bearing capacity. The system will automatically obtain the anthropometric measurements of the user such as the height, weight, and waist circumference, and the weight of the backpack. This innovation will provide solution to the negative effects of backpack overloading by implementing a technology that could be designed and implemented to diminish the negative effects of excessive weight of upper extremity load and thus adapt ways to be able to alert the user with his maximum weight bearing capacity.

\section{EXPERIMENTAL}

\section{System hardware}

A lay-out of the intelligent backpack system is shown in Fig. 1. The prototype was made from an existing frame of a hiking backpack which was then reinforced to fit the desired prototype. It was composed of a three-division compartment with two wide padded shoulder straps. It also had a devised waist belt, which had a limit switch and a series of resistors to measure the waist circumference of the user.

Weight sensor. The weight sensor that was used in the study is a Flexiforce A201 force sensor as displayed on Fig. 2. In a circuit, it acts as a force sensing resistor. The application of the force to

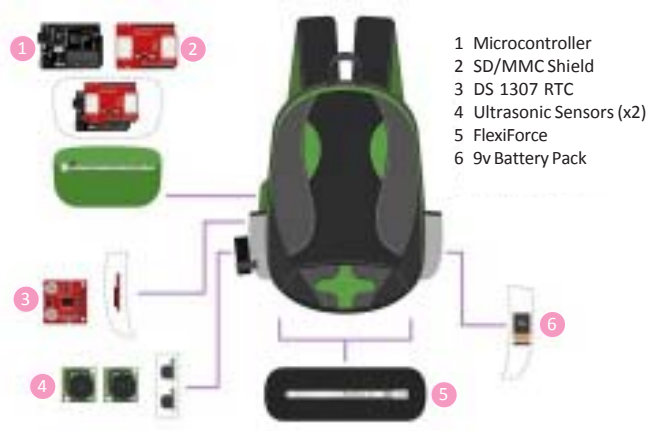

Figure 1. Backpack layout

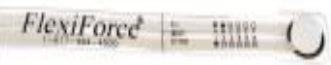

Figure 2. Flexiforce A201 sensor [8] 
the active sensing area would cause to a change in resistance of the sensing element in inverse proportion to the force applied. Figure 3 shows the variation of the sensor resistance with the weight load. A puck, or shim, was placed on the sensing area to concentrate and ensure that it captures the applied load whether its surface is larger than the sensor diameter.

The force sensor into an application was incorporated to a force-to-voltage circuit (Fig. 4). A $5.1 \mathrm{k} \Omega$ gave the most precise sensitivity of the sensor. A fixed resistor of $100 \mathrm{k} \Omega$ was also used to ample the voltage divider circuit.

Figure 5 displays the actual setup of the Flexiforce sensor on a devised base. The base is designed such that its size is fitted at the bottom of the backpack. The base is also equipped with soft flexible springs to

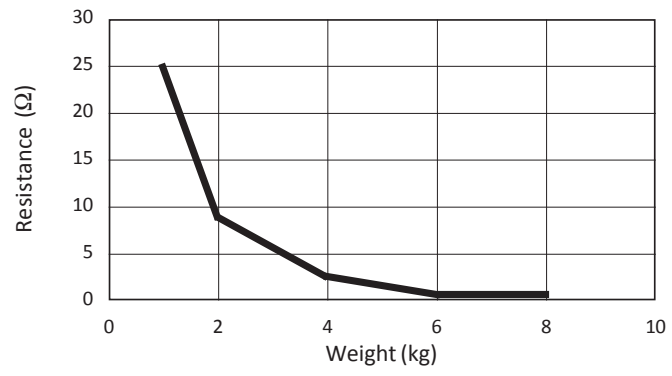

Figure 3. Calibration curve of weight sensor (Flexiforce A201)

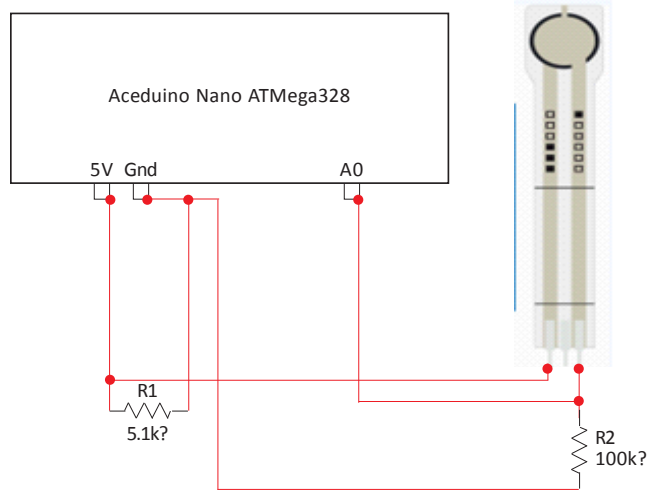

Figure 4. Schematic circuit for the Flexiforce sensor concentrate the load on the Flexiforce itself and to allow precise measurement

Ultrasonic sensor. A US-100 ultrasonic sensor was incorporated in the system to measure the distance from the bottom of the backpack to the ground as, as a requirement to be used in estimation of the height of the user. The sensor assesses features of a target by interpreting the echoes from transmitted sound waves. It establishes the distance of the object through the time interval as the signal was sent as to its echo had received. Figure 6 presents the location of the ultrasonic sensor, in actual.

Devised waist belt system. A waist belt system was devised to measure the waist circumference of the user. This data is necessary to approximate the weight of the user [9]. The system is based on the ability of the system to generate several

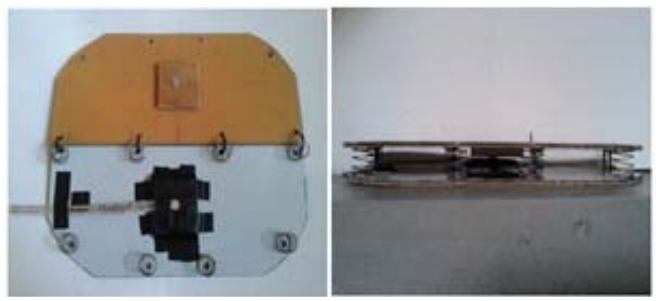

Figure 5. Actual setup of the Flexiforce A201 sensor on the base of the backpack

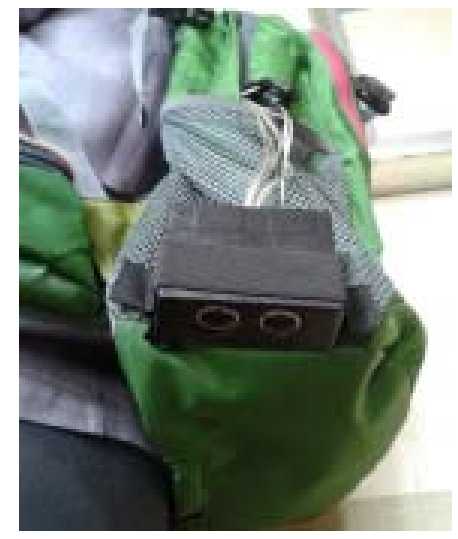

Figure 6. Location of the ultrasonic sensor 
predetermined waist measurements using an array of resistors.

It involves a series of $1 \mathrm{~K}$ ohm resistors placed on the belt as shown in Fig. 7. Every resistor was positioned adjacent to each other with a constant displacement of $20 \mathrm{~mm}$ in between. The gaps correspond to a fixed adjustment that fits the waist circumference of the user.

Once the movable/adjustable strap is engaged to a certain position, the corresponding resistor is neutralized and sends open circuit signal to the microprocessor. Each resistor corresponds to a waist measurement.

Limit switch. A limit switch was integrated in the buckle of the belt system for the measurement of the waist circumference of the user. It is a type of sensor that cut off power automatically at or near the limit of travel of a moving object controlled by any means. This sensor is operational when there is a contact on an actuator of the switch that is mounted on a

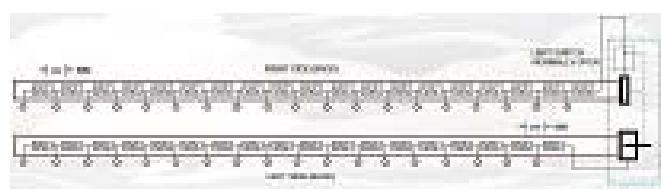

Figure 7. Series of resistors with limit switch for the belt system

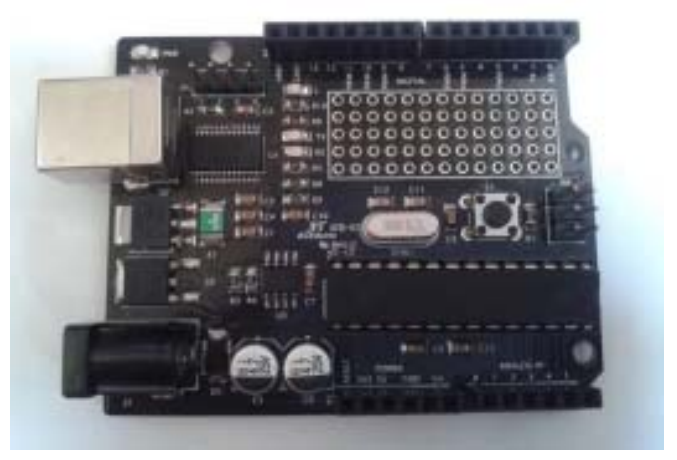

Figure 8. Aceduino ATmega328 microcontroller remote location which can cause the sensor being actuated by an object other that human operation.

Microcontroller. All of the sensors were interfaced into a microcontroller which is programmed to store the acquired data to the memory card for the database system. It is also exploited to compute all of the needed values as the algorithm to be followed were also etched into it. The microcontroller that was used in this study is Aceduino ATmega328 as displayed on Fig. 8.

\section{System operation scheme}

The schema of the system operation is presented on Fig. 9. The initialization of the variables to be used on all of the consequent procedures are obtained under the input pre-processing stage. Gathered data from the sensors were assessed to equip in the preparation for processing.

Calculation of the adequate weight of the backpack will be executed subsequent to the

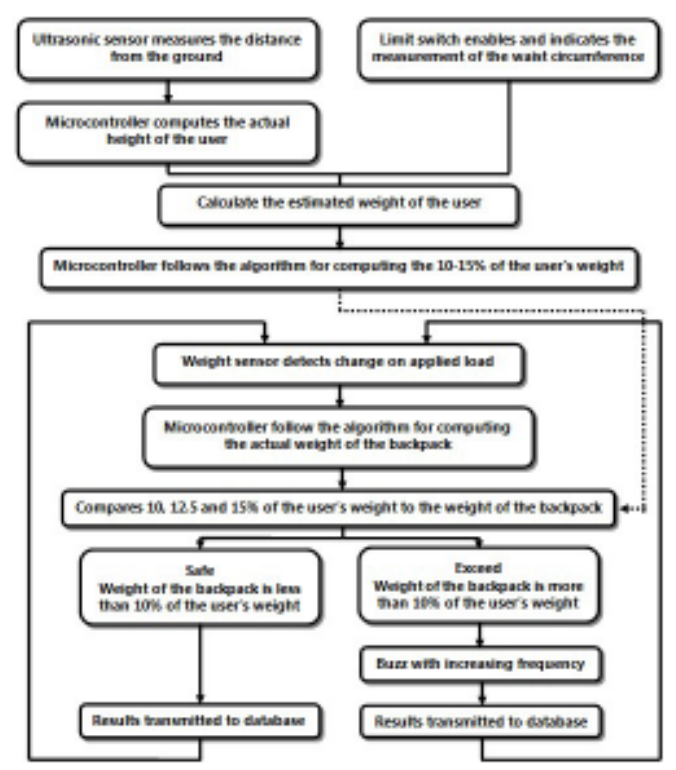

Figure 9. General overview of how the overall system works 
preparation of the variables to be utilized in the process. Given that the approximation of the body weight of the user can be determined, the equivalent $12.5 \%$ of its body weight can be attained. This will search out the maximum capacity of the body of an individual that he ought to carry. Moreover, it represents the utmost value under a tolerance of $\pm 2.5 \%$ that is still acceptable for the user as the weight of the load.

Evaluation whether the backpack is at acceptable weight was implemented through the comparison between the actual weight of the backpack detected by the sensor and the calculated acceptable load for the user consequently. In this process, it will determine the safe load carriage for an individual and set it out directly to the output through a feedback.

If the load is unsafe or has exceeded the threshold for maximum capacity that an individual should carry, it has a buzz like sound that has a continuous increasing frequency to convey awareness to the user. Furthermore, there is a database that will serve as the monitoring means for the weight of the backpack and the user's height, weight and waist circumference, through a memory card. There will only be a transmission of data onto the database only if the weight sensor will detect a substantial change in load.

Modelling. To process both the linear and multiple regressions in this study, the Analysis ToolPak add-in in Excel is utilized. There are three models developed corresponding to the prediction of the height of the user, weight of the user, and the weight of the backpack load.

Model for backpack load. Equation 1 expressed the linear regression model of Load on the voltage measured by the Flexiforce sensor in the following form:
Load $=0.467819849 \times$ Flexiforce +2.006886307

where the variable Flexiforce represents the voltage measured in accordance to the load inside the backpack.

The linear regression model derived from the voltage measured by the Flexiforce sensor yielded a predicted backpack load for every weight applied with a range of 1.5-9 kg. Interpreting the equation, for each unit increase in the Flexiforce sensor value, the voltage increases with 0.467819849 units. This equation is then used to do a forecast in the backpack load using sensor value measured by the Flexiforce.

Model for user's height. Equation 2 expressed the linear regression model of height on the distance measured by the ultrasonic sensor in the following form:

$$
\begin{aligned}
& \text { Height }=1.495935171 \times \text { Ultrasonic }+ \\
& \quad 32.94391256
\end{aligned}
$$

where the variable Ultrasonic represents the distance measured from the bottom of the backpack to ground.

The linear regression model derived from the distance measured by the ultrasonic sensor yielded a predicted user's height for every individual respondent. Interpreting the equation, for each unit increase in the ultrasonic distance, the user's height increases with 1.495935171 units. This equation is then used to do a forecast in the user's height using whatever distance measured by the ultrasonic sensor.

Model for user's weight. Equation 3 expressed the multiple regression model of the user's weight on the height of the user and on the waist circumference of the user in the following form: 
Weight $=1.540038776 \times$ Height +1.279653512

$\times$ Waist -225.3635219

where the variable Height represents the height of the user and the waist represents the waist circumference of the user.

The multiple regression model derived from the height and waist circumference yielded a predicted weight for every individual respondent with age between $10-24$ years old. Interpreting the equation, for each unit increase in the height, the user's weight increases with 1.540038776 units, and for each unit increase in waist circumference, the user's weight increases with 1.279653512 units. This equation is then used to do a forecast in the user's weight using whatever height and waist circumference measured by the ultrasonic sensor and belt system, respectively.

\section{RESUlTS AND DISCUSSION}

In order to check the accuracy of the weight alert backpack, measurements were carried out on a total of 120 respondents ranging from 10 to 24 years old; 62 were males and 58 were females. Their anthropometric measurements such as their weight, height, and waist circumference are first obtained. After which, they are asked to wear the backpack and the respondents carried a test weight of $3 \mathrm{~kg}$, and their corresponding $10 \%, 12.5 \%$, and $15 \%$ of their body weight. The proponents monitored whether the alert system goes off whenever the $10 \%$ of the weight of the user or a weight more than that is reached. A "hit" is achieved when the alarm triggered, otherwise, it is “miss”.

Table 1 shows the summary of the audio response wherein $84.17 \%$, $85 \%$, and $80.83 \%$ of the tests indicate that the system was able to give off an alarm at the stage where the load of the backpack is at $10 \%, 12.5 \%$ or $15 \%$ of the user's body weight, respectively.

Table 2 shows the ranges of percent differences for the different backpack loads such as the test weight, and the corresponding $10 \%, 12.5 \%$, and $15 \%$ of the user's body weight. In general, the ranges of percent difference are from around $0 \%$ up to $10 \%$.

Table 3 shows the ranges of percent differences for the anthropometric measurements such as the weight, height, and waist circumference of the user. In general, the ranges of percent difference are again from around 0-10\%.

Percent differences for the different backpack loads and for the anthropometric measurements are also computed. The measured values coming from the sensors and the theoretical values are compared in this part. It is observed that only

Table 1. Audio Response DATA

\begin{tabular}{l|c|c|c}
\hline \multicolumn{4}{c}{ Alert System } \\
\hline & $\begin{array}{c}\text { Alert goes } \\
\text { off at 10\% } \\
\text { of user's } \\
\text { body } \\
\text { weight }\end{array}$ & $\begin{array}{c}\text { Alert goes } \\
\text { off at } \\
12.5 \% \text { of } \\
\text { user's } \\
\text { body } \\
\text { weight }\end{array}$ & $\begin{array}{c}\text { Alert goes } \\
\text { off at 15\% } \\
\text { of user's } \\
\text { body } \\
\text { weight }\end{array}$ \\
\hline Hit & 101 & 102 & 97 \\
\hline Miss & 19 & 18 & 23 \\
\hline Potal & 120 & 120 & 120 \\
\hline
\end{tabular}

Table 2. Ranges of Percent Differences for the Different Backpack Loads

\begin{tabular}{l|c|c}
\hline \multicolumn{3}{c}{ Ranges of Percent Differences for the Backpack } \\
Loads
\end{tabular}

Table 3. Ranges of Percent Differences for the Anthropometric Measurements

\begin{tabular}{|c|c|c|}
\hline \multicolumn{3}{|c|}{$\begin{array}{c}\text { Ranges of Percent Difference for the } \\
\text { Anthropometric Measurements }\end{array}$} \\
\hline & Minimum & Maximum \\
\hline Weight & $0.03 \%$ & $9.70 \%$ \\
\hline Height & $0.01 \%$ & $10.20 \%$ \\
\hline Waist Circumference & $0 \%$ & $9.52 \%$ \\
\hline
\end{tabular}


minimal values of percentage error are present on both cases.

For the test weight of $3 \mathrm{~kg}$, the percent difference ranges from $0.33 \%$ to $10.67 \%$. For a backpack load corresponding to $10 \%$ of the user's body weight, the percent difference ranges from $0 \%$ to $8.33 \%$. For a backpack load corresponding to $12.5 \%$ of the user's body weight, the percent difference ranges from $0.06 \%$ to $10.51 \%$. And for a backpack load corresponding to $15 \%$ of the user's body weight, the percent difference ranges from $0.09 \%$ to $10.10 \%$.

For the user's weight, the percent difference ranges from $0.03 \%$ to $9.70 \%$. For the user's height, the percent differences ranges from $0.01 \%$ to $10.20 \%$. And for the user's waist circumference, the percent difference ranges from $0 \%$ to $9.52 \%$. Having minimal values of percentage error implies that the system embedded on the backpack will be able to provide weights close to the actual load being carried by the user. In addition, the weight, height, and waist circumference will be closely approximated with the actual measurements of the user.

For the database monitoring part, Fig. 10 displays a sample of a content of a .txt file saved in an SD memory card. The database stores details involving the date and time the

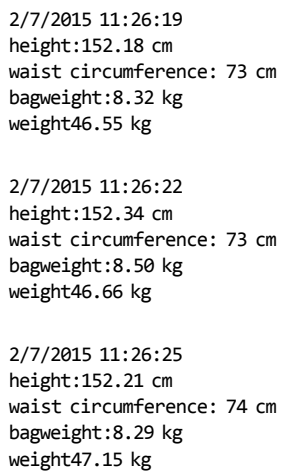

Figure 10. Sample content of the database measurement is obtained, and the instantaneous anthropometric measurements of the user and the corresponding bag weight he is carrying.

\section{Conclusion}

A backpack that can automatically approximate the weight of the user in order to determine the precise weight of the load appropriate for the body by establishing the maximum weight bearing capacity has been successfully designed and implemented.

\section{REFERENCES}

[1] Horovitz B. August 2007. [Online]. Available: http:/ /abcnews.go.com/.

[2] Zielonka J. Why Your Child Needs a "Backpack Buddy" 2011.

[3] P.P.Society. Backpacks and Children. Philippine Pediatric Society Policy Statements 2006; 1(3):11-16.

[4] Don't Let Backpacks Get You Down. Children's Healthcare of Atlanta 2009.

[5] Luttman A, Jager M, \& Griefahn B. Preventing Muscoloskeletal Disorders in the Workplace. Protecting Workers' Health Series 2003; 5:132.

[6] Ramprasad M, Alias J, \& Raghuveer A. Effect of Backpack Weight on Postural Angles in Preadolescent Children. Indian Pediatrics 2010 July; 47:575-580.

[7] Ross A. System and Related Methods for Preventing Back Injury. (United States of America Patent 7404506, 2008 July 29).

[8] Tekscan. Flexiforce Sensors User Manual. (South Boston Massachusetts: Tekscan, Inc., 2010).

[9] Training Manual No. 1: Limit Switches - 101. 1206 Hatton Road. 